\title{
Loss of Dicer fowls up centromeres
}

\author{
Sharon A. White and Robin C. Allshire \\ Centromeres, specialized regions on chromosomes, are essential for accurate chromosome segregation during cell \\ division. In fission yeast, the RNA interference machinery has a pivotal function in the assembly of centromeric \\ heterochromatin, which mediates sister centromere cohesion. Studies in vertebrate cells now suggest that many \\ aspects of this process are conserved.
}

RNA interference (RNAi) is an evolutionarily conserved mechanism whereby doublestranded RNAs (dsRNAs) target homologous transcripts for degradation. Long dsRNAs are cleaved by the endonuclease Dicer to produce small interfering RNAs (siRNAs) of around 21 nucleotides in length. These siRNAs become incorporated into the RNA-induced silencing complex (RISC), which directs them to their homologous RNA target. RNAi functions in several cellular processes, such as gene silencing, protection of genome stability from viruses and transposable elements ${ }^{1}$ and the assembly of silent chromatin, including heterochromatin at centromeres ${ }^{1-3}$.

Centromeres are sites on chromosomes where the kinetochore complex assembles and mediates interactions with spindle microtubules, thus ensuring accurate chromosome segregation. In many eukaryotes, the kinetochore is embedded in heterochromatin ${ }^{4}$. Human centromeric DNA is primarily composed of $\alpha$-satellite repeats arranged in tandem arrays of $1,000-5,000 \mathrm{~kb}^{4}$. Arrays of perfect alphoid repeat DNA provide a good substrate for the de novo assembly of active centromeres. This correlates with the presence of intact binding sites for CENP-B, an $\alpha$-satellite-binding protein ${ }^{5}$. Remarkably, human centromeres maintain their activity when transferred into chicken DT40 cells by somatic cell fusion to form hybrid cell lines. This suggests that host chicken centromere proteins can recognize and propagate a pre-existing kinetochore on introduced human chromosomes 4 .

In fission yeast, the assembly of heterochromatin at centromeres is vital for tight physical cohesion between sister centromeres, and is therefore critical for accurate chromosome segregation ${ }^{6}$. Observations in metazoans also suggest a link between heterochromatin formation and sister chromatid cohesion ${ }^{6}$. Evidence in fission yeast, plants and flies sug-

Sharon A. White and Robin C. Allshire are at The Wellcome Trust Centre for Cell Biology, The Michael Swann Building, University of

Edinburgh, Edinburgh EH9 3JR, UK.

e-mail:sharon.a.white@ed.ac.ukand

robin.allshire@ed.ac.uk

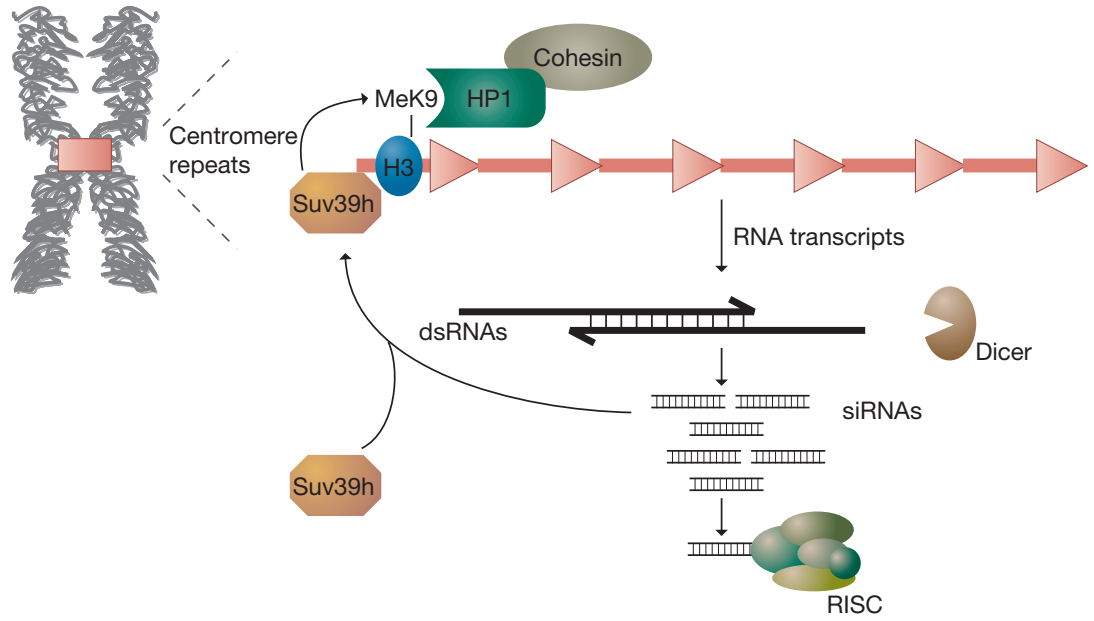

Figure 1. A model for RNAi-mediated heterochromatin assembly in vertebrates. Centromeric non-coding transcripts form dsRNAs that are processed by Dicer to produce siRNAs. Incorporation of siRNAs into RISC recruits the histone methyltransferase Suv39h, which methylates histone $\mathrm{H} 3-\mathrm{K} 9$ resulting in HP1 binding. The formation of heterochromatin results in recruitment of cohesin and ensures accurate chromosome segregation.

gests that siRNAs function to recruit histoneH3-Lys-9 methyltransferases, and DNA methyltransferases, to specific loci to direct the chromatin- and DNA modifications required for heterochromatin formation $^{1-3,7-9}$. In fission yeast and in flies, mutations in components of the RNAi pathway result in defective heterochromatin as seen by loss of silencing, loss of specific histone methylation and delocalization of the heterochromatin-associated proteins HP1 or Swi6 (refs 2, 3, 7-11). In fission yeast, defects in the RNAi pathway also cause loss of Rad21 and thus cohesion at centromeres, resulting in aberrant chromosome segregation ${ }^{5,10,11}$.

It is not known whether RNAi mediates the assembly of centromeric heterochromatin in vertebrates. On page 784 of this issue ${ }^{12}$, Fukagawa and colleagues demonstrate that the link between RNAi, heterochromatin formation and centromeric cohesion is conserved in vertebrates. They show that a conditional lossof-function mutant of Dicer in chicken DT40 cells causes defects in heterochromatin formation and chromosome segregation, similar to those observed in fission yeast. Depletion of Dicer ultimately results in cell death, with an accumulation of abnormal mitotic cells.

Previous analyses demonstrated that a heterochromatin-related structure at mammalian centromeres is RNase sensitive, consistent with a role for RNA in heterochromatin formation ${ }^{13}$. In agreement with this idea, non-coding transcripts homologous to centromeric repetitive DNA elements have been observed in both mouse embryonic fibroblasts and fission yeast $^{2,14}$. In their studies, the authors used a chicken DT40 hybrid cell line carrying human chromosome 21. Consistent with observations in other systems, transcripts homologous to the centromeric repeats of human chromosome 21 clearly accumulate in Dicer-deficient DT40 cells. Extremely low levels of these human centromeric transcripts are also seen in Dicer $^{+}$hybrid cells, in accordance with data from fission yeast suggesting that centromeric transcripts are rapidly turned over in wild-type cells $^{12}$. As chicken centromeric DNA has not been characterized, the authors were unable to test if transcripts are also produced from endogenous chicken centromeres.

In fission yeast, centromeric transcripts are processed into siRNAs by the RNAi pathway. The detection of short RNAs $(\sim 30$ 
nucleotides) homologous to human centromeric $\alpha$-satellite repeat DNA in Dicer $^{+}$ cells, which decline in Dicer-deficient cells, suggests that Dicer and the RNAi machinery are required to cleave these human centromere repeat transcripts. However, these short RNAs persist to some extent in Dicer-depleted cells, perhaps due to some residual Dicer activity ${ }^{12}$.

Fission yeast centromeres contain a clearly demarcated central domain where kinetochores assemble, which is flanked by heterochromatin ${ }^{4}$. In contrast, vertebrate centromeres lack a clear boundary between heterochromatin and the CENP-A-kinetochore-specific chromatin that marks the site of kinetochore assembly ${ }^{4}$. Therefore, it is possible that centromeric $\alpha$-satellite transcripts in vertebrate cells have a function in kinetochore assembly. Perhaps only a subset of the $\alpha$-satellite array repeats are transcribed, triggering patches of heterochromatin assembly with kinetochore proteins being loaded in between; but in this scenario it is unclear how the boundaries for this would be established and maintained. Regardless, the localization of the kinetochore-specific proteins CENP-A and CENP-C seems to be normal in Dicer-deficient cells, suggesting that defective RNAi does not completely disrupt kinetochore assembly and that the presence of kinetochore proteins does not impede transcription of the centromeric repeats in vertebrate cells. However, the authors found that heterochromatin formation is perturbed in Dicer-deficient cells. In Dicer ${ }^{+}$cells, HP1 proteins colocalize to discrete nuclear foci in the vicinity of CENP-C at both endogenous and human centromeric heterochromatin. In contrast, although HP1 signals are still detectable in Dicer-deficient cells, HP1 becomes diffusely distributed and seems to associate non-specifically with all chromatin, similar to the redistribution of HP1 and HP2 observed in RNAi fly mutants ${ }^{3}$. It is probable that specific histone modifications associated with centromeric heterochromatin, such as H3-K9 and H4-K20 methylation, are also disrupted, but this was not tested.

Further analyses revealed that the Rad 21 cohesin does not concentrate at endogenous or human chromosome 21 centromeres, but displays a more dispersed localization in Dicer-deficient cells. This suggests that Dicerdependent heterochromatin is required for cohesion between sister centromeres, as found in fission yeast ${ }^{5,10,11}$. Consistent with this, these cells exhibit premature sister centromere separation and arrest in mitosis. As HP1 localization and cohesion seems to be defective at all centromeres in these DT40 Dicer-deficient cells, this suggests that RNAi is also required for heterochromatin assembly and cohesion at endogenous chicken centromeres ${ }^{12}$.

The data presented in this study suggest a general model for heterochromatin formation, and suggest that RNAi-mediated heterochromatin assembly has a direct and conserved role in recruiting cohesin (Fig. 1). Surprisingly, the budding yeast genome does not encode any known RNAi components and it also lacks centromeric heterochromatin, although cohesin is enriched around centromeres ${ }^{6}$. So why is RNAi-dependent heterochromatin required in higher eukaryotes? In metazoans, cohesin is removed from along chromosome arms early in mitosis. It is possible that heterochromatin functions in the protection of centromeric cohesin from this removal process until it is cleaved at anaphase ${ }^{6}$. However, there must be other roles for this centromeric heterochromatin as, similarly to budding yeast, all cohesion between sister chromatids is lost simultaneously at anaphase in fission yeast. Centromeres in fission yeast, plants and animal cells in mitosis are contacted by multiple spindle microtubules, whereas in budding yeast, mitotic centromeres only attach to one spindle fibre. Therefore, it is possible that centromeric heterochromatin has a structural role in organising and orienting mutiple microtubule-binding sites at sister centromeres ${ }^{4}$. In this case, RNAi-dependent chromatin modifications would probably be required for the formation of such a structure.

This and other studies raise many questions. First, it is unclear precisely how siRNA nucleates heterochromatin assembly. Observations in plants suggest that siRNA may participate in RNA-DNA interactions that result in chromatin modifications. Alternatively, it is possible that nascent chromatin-associated transcripts are targeted by siRNAs and a RISClike complex in the nucleus to form heterochromatin. Interestingly, in plants, the silent state can be propagated in the absence of a dsRNA trigger, suggesting that RNAi functions to nucleate and establish silent chromatin, and also that it can be subsequently propagated by chromatin-associated factors ${ }^{15}$. Second, how often centromere repeats are transcribed is not known. They may be transcribed occasionally in a stochastic manner, allowing re-establishment of heterochromatin when required, or, transcription may occur at a particular stage of the cell cycle allowing reformation of robust heterochromatin in conjunction with, or following, replication. Finally, exactly how centromere repeats are transcribed, and whether transcription occurs across the entire centromere repeat region, is not known. It is possible that centromere-repeat-binding proteins such as CENP-B promote repeat transcription, but this and other issues await further investigation.

The ablation of Dicer function in chicken cells underscores the conserved role of RNAi in heterochromatin assembly and centromere function. Further analyses may ruffle feathers regarding how RNAi promotes heterochromatin assembly.

1. Hannon, G. J. Nature 418, 244-251 (2002).

2. Volpe, T. A. et al. Science 297, 1833-1837 (2002).

3. Pal-Bhadra, M. et al. Science 303, 669-672 (2004).

4. Sullivan, B. A., Blower, M. D. \& Karpen, G. H. Nature Rev. Genet. 2, 584-596 (2001).

5. Ikeno, M. et al. Nature Biotechnol. 16, 431-439 (1998).

6. Bernard, P. \& Allshire, R. Trends Cell Biol. 12, 419-424 (2002)

7. Schramke, V. \& Allshire, R. Science 301, 1069-1074 (2003).

8. Verdel, A. et al. Science 303, 672-676 (2004).

9. Schramke, V. \& Allshire, R. Curr. Opin. Genet. Dev. 14, 174-180 (2004).

10. Volpe, T. et al. Chromosome Res. 11, 137-146 (2003).

11. Hall, I. M., Noma, K. \& Grewal, S. I. Proc. Natl Acad. Sci. USA 100, 193-198 (2003).

12. Fukagawa, T. et al. Nature Cell Biol. 6, 784-791 (2004).

13. Maison, C. et al. Nature Genet. 30, 329-334 (2002).

14. Lehnertz, B. et al. Curr. Biol. 13, 1192-1200 (2003).

15. Jones, L., Ratcliff, F. \& Baulcombe, D. C. Curr. Biol. 11, 747-757 (2001). 